\title{
1 Effects of tidal flushing regimes on mangrove roots receiving 2 wastewater contaminated with PAHs and PBDEs
}

3

$4 \quad$ N. $\mathrm{Pi}^{\mathrm{a}, \mathrm{b}}$, Y. Wu ${ }^{\mathrm{a}}$, H.W. Zhu ${ }^{\mathrm{a}}$, Y.S. Wong ${ }^{\mathrm{c}}$ and N. F. Y. Tam ${ }^{\mathrm{a}, \mathrm{d}^{*}}$

$6 \quad{ }^{\text {a }}$ Department of Biology \& Chemistry, City University of Hong Kong, Hong Kong

7 SAR, China

8 b NUS Environmental Research Institute, National University of Singapore,

9 Singapore

c School of Science and Technology, Open University of Hong Kong, Hong Kong

11 SAR, China

d State Key Laboratory in Marine Pollution, City University of Hong Kong, Hong

Kong SAR, China

14

*Corresponding Author

Address: Department of Biology \& Chemistry, City University of Hong Kong, Tat

Telephone: +852-3442-7793; Fax: +852-3442-0552

Email address: bhntam@cityu.edu.hk 


\section{Abstract}

The effects of tidal flushing regimes on roots of wastewater-stressed mangrove plants were unknown. The present study showed that patterns of radial oxygen loss (ROL) in two mangrove species, Excoecaria agallocha and Kandelia obovata, receiving wastewater containing polycyclic aromatic hydrocarbons (PAHs) and polybrominated diphenyl ethers (PBDEs), were not affected by tidal flushing regimes. However, more ROL was induced under less frequent tidal flushing regimes, and produced along a root of K. obovata than that of E. agallocha. The iron (Fe) plaque formed on roots were negatively correlated to ROL, suggesting that Fe plaque functioned as a 'barrier' to prevent ROL, with stronger effects under less frequent tidal flushing regimes and in $K$. obovata with weaker outer layers. These results revealed that PAHs- and PBDEs-stressed $K$. obovata was more sensitive to tidal flushing regimes than E. agallocha, and long-term exposure would lead to root damage and even plant death.

Key works: Fe plaque, mangrove plants, polycyclic aromatic hydrocarbons, polybrominated diphenyl ethers, radial oxygen loss 


\section{Introduction}

Mangrove wetlands distributed along coastal areas are close to human activities and are subject to anthropogenic contamination. Polycyclic aromatic hydrocarbons (PAHs) and polybromodiphenyl ethers (PBDEs) are two types of persistent organic pollutants commonly found in industrial discharges and in environments. PAHs, toxic, mutagenic and carcinogenic, are resistant to biodegradation, especially in anaerobic sediments where serious accumulation of PAHs can lead to significant adverse health effects (Kanaly and Harayama, 2010; Toyama et al., 2011). PBDEs used as additive flame retardants in a wide range of industrial production are also highly lipophilic and bio-magnified in organisms through food chains and food webs (Ikonomou et al., 2002; Kelly et al., 2008). PBDEs cause various toxic effects to animals such as developmental neurotoxicity and thyroid hormone disruption (Branchi et al., 2002;

Darnerud, 2003). It is common to find that mangrove plants continuously receive anthropogenic PAHs and/or PBDEs from wastewater discharges, as these pollutants have been detected in mangrove ecosystems around the world, such as Hong Kong SAR of China (Ke et al., 2005; Li et al., 2009; Zhu et al., 2014), Brazil (Farias et al., 2008), Mainland China (Cao et al., 2009), Indonesia (Dsikowitzky et al., 2011), India (Binelli et al., 2008) and Senegal (Bodin et al., 2011).

Being intertidal, mangrove plants are subject to tidal flushing, leading to alternating aerobic and anaerobic conditions, and different oxygen content in soil. Mangrove plants had a large ability to release oxygen from root aerenchyma air 
spaces to rhizosphere, a phenomenon called radial oxygen loss (ROL), and form iron (Fe) plaque on root surfaces (Pi et al., 2010a, b, 2011). Not only adapt to waterlogged soil, ROL and Fe plaque also protect wetland plants against toxicants and environmental stresses (Armstrong, 1979; Brix, 1997; Visser et al., 2000; Pi et al., 2009). The patterns and rates of ROL, as well as the concentration of Fe plaque formed on plant roots, were profoundly affected by tidal flushing regimes and pollutants (Moller and Sand-Jensen, 2008; Pi et al., 2010a, b, 2011). However, how tidal flushing affects ROL and Fe plaque in mangrove plants continously receive toxic pollutants is still unknown.

In Hong Kong, mangrove wetlands are mostly inundated by incoming tides twice a day, with the largest tidal range at high spring tides of about $2.8 \mathrm{~m}$ (Tam and Wong, 2000). The mangrove plants distributed in different tidal positions from seaward to landward are exposed to different frequencies of tidal flooding, depending on their tidal zonation. Kandelia obovata, the most common mangrove plant species found in all tidal positions in Hong Kong, are subject to various tidal regimes with different frequencies, while the plants grown in seaward area such as Aegiceras corniculatum and Acanthus ilicifolius are often flooded twice a day, on the contrary, those grown at the very landward location such as Excoecaria agallocha and Heritiera littoralis are only flooded twice a month or even never flooded (Tam and Wong, 2000; Pi et al., 2009). It is logical to hypothesize the responses of stressed mangrove plants to tidal flushing regimes vary among plant species at different tidal positions. 
The present study therefore aims to investigate the effects of tidal flushing regimes on the growth, ROL, formation of Fe plaque, and relationships between ROL and Fe plaque formation, of mangrove plants continuously received wastewater containing different congeners of PAHs and PBDEs. Two mangrove plant species, namely Excoecaria agallocha L. and Kandelia obovata Sheue, Liu \& Yong, were selected, because they distribute in different tidal positions and have different root structures (Pi et al., 2009). Pi et al. also found that K. obovata had larger aerenchyma air spaces to transport $\mathrm{O}_{2}$ within root and was more tolerant to waterlogging, while E. agallocha having stronger outer layers to protect its root against soil toxic pollutants was more tolerant to wastewater ( $\mathrm{Pi}$ et al., 2009). This is the first study comparing the root responses of these two species, under the stress of mixed PAHs and PBDEs, to different tidal flushing regimes.

\section{Materials and methods}

\subsection{Preparation of one-year old seedlings}

One year prior to this study, the propagules of E. agallocha and K. obovata were collected from local mangrove swamps in Hong Kong, germinated and grown in small pots with one propagule per pot. Each small pot had a dimension of $9.6 \mathrm{~cm}$ in diameter and $12.2 \mathrm{~cm}$ in height (total volume was $882.6 \mathrm{~cm}^{3}$ ), and contained surface soil collected from a typical mangrove swamp in Hong Kong with the soil properties described by Pi et al. (Pi et al., 2010a). The pots were placed in a greenhouse with a 
light intensity of $57.29 \mu \mathrm{mol} \mathrm{m} \mathrm{m}^{-2}$, average temperature and humidity of $27^{\circ} \mathrm{C}$ and 70\%, respectively. Every morning, the pots were irrigated with tap water to ensure that the soil was wet but without any water flooded on soil surface and water was gradually evaporated during the day. During the one-year growing period, no nutrients or salts were added as propagules stored enough nutrients for germination and initial growth. Ye and Tam also concluded that nutrients in mangrove soil were sufficient for subsequent growth and the mangrove seedlings in Hong Kong grew better at low salinity (Ye and Tam, 2002). The one-year seedlings were healthy with green leaves.

\subsection{Experimental design}

A total of 48 pots, each with a dimension of $12.4 \mathrm{~cm}$ in diameter, $10.1 \mathrm{~cm}$ in height and total volume of $998.4 \mathrm{~cm}^{3}$, filled with the same mangrove soil as described above to a depth of $9 \mathrm{~cm}$, were prepared. Half of the pots were planted with E. agallocha and the other half with $K$. obovata, with one seedling obtained from the above section per pot. The transplanted seedling was allowed to acclimate in the new pot condition and was irrigated with tap water every morning to keep the soil moist for one month. After acclimation, the pots having the same plant species were further divided into four groups with different tidal flushing regimes: (i) everyday flushing (Te), (ii) oneday with tidal flushing and one-day without (To), (iii) two days with tidal flushing per month (on the $1^{\text {st }}$ and $15^{\text {th }}$ day, Tt) and (iv) no tidal flushing (control, Tn), with three 
replicates per group. Every day, each pot was irrigated with artificial wastewater containing three compounds of polycyclic aromatic hydrocarbons (PAHs), that is, phenanthrene (Phe), pyrene (Pyr) and benzo(a)pyrene (BaP), and six congeners of polybrominated diphenyl ethers (PBDEs), that is, BDE-47, -100, -99, -154, -153 and 209 during the exposure period (at about 15:00). The concentrations of Phe, Pyr, BaP, BDE-47, $-100,-99,-154,-153$ and -209 in the artificially prepared wastewater were 870.00, 135.90, 9.20, 10.94, 3.34, 16.86, 1.21, 1.56 and $18.77 \mu \mathrm{g} \mathrm{L}{ }^{-1}$, respectively.

The concentrations of PAHs and PBDEs in the artificial wastewater in the present study were chosen based on the following considerations: 1) the water solubility of each contaminant based on the Estimation Programs Interface (EPI) Suite TM (developed by the US Environmental Protection Agency's Office of Pollution Prevention and Toxics and Syracuse Research Corporation) estimated from Log Kow at $25^{\circ} \mathrm{C}$ and fragments, as well as the real measurement in our greenhouse; 2) the levels of PAHs and PBDEs in raw wastewater reported in previous studies ranged from few $n g \mathrm{~L}^{-1}$ to tens $\mathrm{mg} \mathrm{L}^{-1}$ (Song et al., 2006; Peng et al., 2009; Sanchez-Avila et al., 2009; Clarke et al., 2010); and 3) the levels of individual PAH and PBDE compound used in present study were their respective highest water solubility detected in our greenhouse condition, comparable to or ten times higher than those in field applications, in order to assess the effects of tidal flushing regimes on mangrove roots in heavily-polluted areas. The three PAHs (Phe, Pyr and BaP) were purchased from Sigma-Aldrich (St. Louis, MO, USA) with purity higher than 96\%, and the six 
PBDE congeners (BDE-47, -100, -99, -154, -153 and -209) were purchased from AccuStandard (New Haven, CT, USA) with purity higher than 99\%. The daily hydraulic loading (the volume of artificial wastewater irrigated to each pot in every tide) was $80 \mathrm{~mL}$ which is equivalent to a hydraulic retention time of 5 days.

To simulate tidal flushing, the artificial seawater, prepared by dissolving commercially available salts in tap water with a salinity of 15 parts per thousands (ppt) was flooded to each pot at a water depth of $4 \mathrm{~cm}$ above soil surface on the tidal flushing day (from 18:00 pm to 10:00 am of the next day, about 16 hours) to simulate high tide, followed by 8 hours low tide by draining seawater from the pot and let the soil exposed and dried out gradually. Seawater was reused during the study. The pots were placed in the same greenhouse as described above and the experiment lasted eight months. Three pots from each group were randomly retrieved at the end of 4and 8-months. The seedling was harvested, ROL, Fe plaque formation on root surface, and biomass of leaf, stem and root were determined.

\subsection{Determination of ROL along a lateral root and biomass}

The rates of ROL at fixed distances behind the tip $(0,2,4,6$ and $8 \mathrm{~cm}$ from the root tip) of the intact lateral root, with lengths ranging from 8 to $12 \mathrm{~cm}$ and approximately $6 \mathrm{~cm}$ below root-shoot junction, were measured in an $\mathrm{O}_{2}$-free root medium using the cylindrical root-sleeving $\mathrm{O}_{2}$ electrode (Armstrong et al., 1994), within 24 hours after harvesting. The detailed procedures were described in Pi et al. (Pi et al., 2010a, b). In 
brief, the intact root system was gently immersed into the well prepared $\mathrm{O}_{2}$-free solution containing $0.5 \mathrm{mM} \mathrm{CaSO}_{4}$ and $5 \mathrm{mM} \mathrm{KCl}$ into agar-water medium $(0.05 \%$ $\mathrm{w} / \mathrm{v}$ ), while the other parts of the plant were in air. The section of the lateral root to be measured was inserted through a cylindrical $\mathrm{O}_{2}$ electrode, and the 'diffusion current' generated by the reduction of oxygen at the polarized platinum surface was recorded, at least two hours after transferring the root into the $\mathrm{O}_{2}$-free solution. All measurements were made at the polarizing voltage of $1 \mathrm{mV}$. The rate of ROL along a root was calculated based on the 'diffusion current' at each position measured and their respective surface area.

After the measurement of ROL, the same lateral root was cut and used for the determination of Fe plaque. The plants were then divided into leaf, stem and root portions, oven-dried at $70^{\circ} \mathrm{C}$ for $48-72$ hours to calculate the oven-dried biomass of each portion. The plant dry biomass of each species was also measured at the beginning of the experiment.

\subsection{Determination of Fe plaque formation on root surfaces}

The lateral root selected was extracted using the cold dithionite-citrate-bicarbonate (DCB) extraction method (Taylor and Crowder, 1983). The detailed procedures were described in Pi et al. (Pi et al., 2010b, 2011). In brief, each collected root section was washed and agitated for three hours in a cold DCB solution consisting of $40 \mathrm{~mL}$ of $0.3 \mathrm{M}$ sodium citrate $\left(\mathrm{Na}_{3} \mathrm{C}_{6} \mathrm{H}_{5} \mathrm{O}_{7} \cdot 2 \mathrm{H}_{2} \mathrm{O}\right), 5 \mathrm{~mL}$ of $1.0 \mathrm{M}$ sodium bicarbonate 
190

191

192

193

194

195

196

197

198

199

200

201

202

203

$\left(\mathrm{NaHCO}_{3}\right)$ and $3 \mathrm{~g}$ of sodium dithionite $\left(\mathrm{Na}_{2} \mathrm{~S}_{2} \mathrm{O}_{4}\right)$. The wash was collected and the resulting solution was made to a volume of $100 \mathrm{~mL}$. Then $20 \mathrm{~mL}$ of the solution was used for the measurement of Fe plaque. The concentrations of $\mathrm{Fe}^{\mathrm{n}^{+}}$were measured with Inductively-Coupled Plasma-Atomic Emission Spectrometry (ICP-AES, PerkinElmer, USA), according to the standard method (Page et al., 1992).

All analytical procedures were subject to strict quality assurance and control. The samples were analyzed within 24 hours after each harvesting. Standard addition method was employed to ensure the accuracy and recovery of the analysis. Five calibration standards (prepared using Trace Metals Standard obtained from AccuStandard, USA), with concentrations of $\mathrm{Fe}^{\mathrm{n}+}$ ranging from 0 to $10 \mu \mathrm{g} \mathrm{mL}^{-1}$, was used for the quantification of $\mathrm{Fe}^{\mathrm{n}+}$ by ICP-AES. Control standard with $\mathrm{Fe}^{\mathrm{n}+}$ concentration at $5 \mu \mathrm{g} \mathrm{mL}{ }^{-1}$ was analyzed for every four samples to ensure instrument performance during analysis. Each sample was repeatedly analyzed for three times, the standard deviation was lower than $1 \%$ and the detection limit was $0.18 \mathrm{ng} \mathrm{mL}^{-1}$. The washed roots were freeze-dried to obtain the dry weight. The concentration of Fe plaque on root surface was calculated according to Taylor and Crowder as follow (Taylor and Crowder, 1983):

[Fe plaque on root surface $]=0.1591 \times\left[\mathrm{Fe}^{\mathrm{n}+}\right]$ in the extraction / root dry weight $(\mathrm{mg}$ $\mathrm{g}^{-1}$ root d.wt).

\subsection{Statistical analyses}



each group were calculated. A parametric analysis of covariance (ANCOVA), with

213 time as the covariate, and species vs. tidal flushing regimes as the two factors, was used to test any differences in plant dry biomass of each individual part, rate of ROL from one section of a root and total rate of ROL along a root, as well as the formation of Fe plaque on root surface. A parametric independent sample T-test was conducted to test any difference between the two sampling times in each tidal flushing regime in each species. The linear regressions between Fe plaque formed (x) and total rate of ROL released (y) were calculated. The differences of the regression coefficients between more (Te, To) and less tidal regime (Tt, Tn) in each plant species were tested following the principles described in Zar (Zar, 1999). Statistical analyses of the ANCOVA test also showed that the dried biomass of leaf, by a parametric independent sample $T$-test. All tests were carried out using the Statistical Package for Social Science (SPSS 16.0 for Windows, SPSS Inc, USA)

\section{Results and Discussions}

\subsection{Plant growth}

All mangrove plants continued to grow during the entire experimental period with continuous discharge of wastewater containing PAHs and PBDEs, but rates of growth were affected by plant species, time and tidal flushing regimes (Fig. 1 and Table S1). stem and root varied significantly between two mangrove species, among tidal 
flushing regimes and between two sampling times (Table 1).The production of leaf, stem and root biomass of E. agallocha was faster from 0- to 4-month, with monthly growth rates varied between 31.85 and $482.56 \%$, while that in second four months (4to 8-month) were lower, between 0 and $47.98 \%$, dependent on plant parts and tidal regimes (Fig. 1 and Table S1). Among different plant parts, temporal changes of stem growth was most significant, with monthly growth rates of more than $200 \%$ in the first four months to less than $20 \%$ in the second four months. Similar pattern was also found in the growth of $K$. obovata, with more rapid growth from the beginning to 4month (monthly growth rates of each plant part varied between 20.85 to $661.44 \%$ ), but plant growth was inhibited from 4-month onward except stem (Fig. 1 and Table S1). Among three plant parts, root growth of K. obovata showed the most significant temporal changes, with more than $200 \%$ growth from 0 - to 4 -month but dropped to negative growth from 4- to 8-month. These results revealed that growth rates differed significantly among plant species and even between plant parts of the same species. The faster growth rate of mangrove plants in the $1^{\text {st }}$ four months than that in the $2^{\text {nd }}$ four months may be due to the accumulation of PAHs and PBDEs in soil caused by the continuous discharge of wastewater, as these contaminants were resistant to biodegradation (Kanaly and Harayama, 2010; Toyama et al., 2011). Although the PAHs in the soil could be utilized as the sole carbon source to support plant growth (Toyama et al., 2011), accumulation of excessive PAHs and PBDEs could cause significant adverse effects to organisms exposed to them (Branchi et al., 2002; 
Darnerud, 2003; Kanaly and Harayama, 2010; Toyama et al., 2011).

Tidal regimes were significant in affecting plant growth but its effect depended on plant species and time (Fig. 1, Table 1 and Table S1). The growth rate of the entire plant of E. agallocha in the first four months increased with decreases in the frequency of tidal regime except Tn (without any tidal flushing); however, the opposite was found from 4- to 8-month with faster growth in Te and To treatments with more frequent tidal flushing (Fig. 1). Different from E. agallocha, the total biomass production of $K$. obovata decreased with the frequency of tidal regime throughout the whole experimental period (Fig. 1). Previous studies also found that the effects of tidal flushing on growth of these two plant species in freshwater without any contamination were different, with $K$. obovata showing positive response to tidal flushing (Cheng et al., 2015), but E. agallocha was not affected by tidal regimes (Pi et al., 2010a, b). The higher tolerance of K. obovata to waterlogging was related to its larger root aerenchyma air spaces for transporting $\mathrm{O}_{2}$ from aerial plant parts to root tips (Pi et al., 2009). However, the present results on growth responses to waterlogging were somewhat deviated from the study by Pi et al. (Pi et al., 2009), variations in root growth rates due to tidal regimes with more flushing frequency were larger in $K$. obovata (decreasing from $661 \%$ at 4 -month to $-10 \%$ at 8 -month with the highest rates in Te and To) than that of E. agallocha throughout the whole experiment (Fig. 1 and Table S1), indicating K. obovata was more sensitive to more frequent tidal flushing than E. agallocha. The continuous discharge of wastewater 
contaminated with PAHs and PBDEs posed pollution stress to plant growth and such stress was more serious when plants were grown in waterlogged soils than that without waterlogging. Waterlogging created more anaerobic conditions in soil leading to the accumulation of toxic pollutants (Ponnamperuma, 1984; Colmer, 2003; Pi et al., 2010b). The "carrier" effects of water to transport pollutants from soil to plants also increased the exposure potential of plant roots to soil pollutants (Colmer, 2003; Pi et al., 2010a). Pi et al. found that E. agallocha developed much stronger root outer layers (epidermis and hypodermis) to protect its roots against soil toxic pollutants than K. obovata (Pi et al., 2009), suggesting that E. agallocha might be more tolerant to toxic pollutants. Among three plant parts, roots appeared to be most sensitive to soil contaminants (Pi et al., 2010a). Therefore, although K. obovata had a higher tolerance to waterlogging than E. agallocha in fresh water conditions (Pi et al., 2009), the weaker root protective outer layers in K. obovata made it more sensitive to tidal regimes under PAHs and PBDEs stresses. The significantly lower monthly growth rates of $K$. obovata from 4- to 8- month than in the first four months (from 0 - to 4month) but such temporal difference was relatively smaller in E. agallocha (Fig. 1 and Table S1) further supported our finding that the latter species was more tolerant to two stresses, waterlogging and pollution.

\subsection{Rates and patterns of radial oxygen loss (ROL) from roots}

The rates of ROL along a lateral root, similar to plant growth, varied significantly 
between mangrove species, tidal flushing regimes and sampling times (Fig. 2 and Table 1). The rates also varied among different sections along a root (Fig. 2). The rates of ROL from roots of E. agallocha and $K$. obovata were the highest in the root tip and declined in the basal and mature zones in seedlings collected from both 4- and 8-month. This indicated that all roots had the same 'tight barrier' pattern to ROL, irrespective to species, sampling time and tidal regimes. Tight barrier ROL is an important adaptation strategy for mangrove plants to flooding (Liu et al., 2009b; Pi et al., 2009). However, higher values in the root tip were found under less frequent tidal flushing regimes ( $\mathrm{Tt}$ and $\mathrm{Tn}$ ) than those under the more frequent tidal flushing regimes, Te and To (Fig. 2). The differences of ROL rates from mature zone, basal zone and root tip of the seedlings subject to $\mathrm{Tt}$ and $\mathrm{Tn}$ were also larger than Te and To. Not only the rates of ROL along a root, the total rates of ROL also significantly affected by mangrove species, tidal flushing regimes and sampling times (Fig. 3 and Table 1). The total rates of ROL decreased from 4- to 8-month in both plant species under all tidal regimes, probably due to the prolonged exposure of wastewater contaminated with PAHs and PBDEs which became toxic, as evidenced by the lower biomass production in the second four months than the first four months (Fig. 1, Table S1), especially $K$. obovata. The toxic and suppression effects of PAHs compounds and PBDEs congeners to mangrove plants were reported (Liu et al., 2009a; Huang et al., 2013; Wang et al., 2014). Similarly, ROL in mangrove seedlings also decreased significantly, especially in apical root zones, when stressed by copper, 
zinc and lead, due to the inhibitory effects of heavy metals on growth, alteration of root permeability and development of aerenchyma tissues (Liu et al., 2009b; Pi et al., 2009; Cheng et al., 2012). Not only mangroves, ROL in various emergent wetland plants was also significantly reduced by the irrigation of strong wastewater containing high concentration of zinc, due to damages to root tissues such as aerenchyma and root porosity by zinc in wastewater (Mei et al., 2014). Jackson et al. reported that wastewater was an environmental stressor changing root properties of in rice (Jackson et al., 1985). Phytotoxins such as organic acids and sulphide also decreased ROL from rice and reeds by altering their root anatomic features (Armstrong and Armstrong, 2005).

Additionally to pollutants, wetland plants change the amount of ROL with time, and temporal variations may be different among species and environmental conditions. The amount of ROL in rice changed during growth, with dramatic increases from tillering to ear emergence and then decreased at the grain-filling stages (Wang et al., 2013). In the present study, more $\mathrm{O}_{2}$ was released from roots of both $E$. agallocha and K. obovata under less frequent tidal flushing regimes ( $\mathrm{Tt}$ and $\mathrm{Tn}$ ) than the two regimes with more frequent tidal flushing (Te and To), the differences in total ROL rates were more obvious at the first four month than that at the second four months and the extent of decrease was larger in Tt and Tn (Fig. 3). Between the two plant species, more $\mathrm{O}_{2}$ was released from the root of $K$. obovata than that of $E$. agallocha, irrespective to sampling time and tidal regimes. The amount of ROL from 
roots was found to increase when there was more $\mathrm{O}_{2}$ demand in immediate root surroundings (Armstrong, 1994; Pi et al., 2010a). ROL is generally known as an adaptation of wetland plants to waterlogging by creating an oxidizing environment, with more ROL under more anaerobic conditions (Armstrong and Armstrong, 2005). However, opposite results were recorded in the present study that the total rates of ROL from roots, same as the changes along the entire root, were significantly lower in Te and To than Tt and Tn treatments (Figs. 2 and 3). This indicated that the pots subject to more frequent tidal flushing had less ROL even though the plants were under longer duration of waterlogging and should have higher $\mathrm{O}_{2}$ demand. The low ROL result may be attributed to the formation of Fe plaque on root surfaces, which was suggested to function as the 'barrier' to prevent ROL according to previous researches (Moller and Sand-Jensen, 2008; Pi et al., 2010b).

\subsection{Formation of Fe plaque on root surface and relationships with ROL}

More Fe plaque formation on root surfaces was found in both E. agallocha and $K$. obovata subject to more frequent tidal flushing ( $\mathrm{Te}$ and $\mathrm{To}$ ) than $\mathrm{Tt}$ and $\mathrm{Tn}$ at the two sampling times (Fig. 4 and Table 1). The concentrations of Fe plaque also increased significantly from 4- to 8-month in both plant species, indicating that the longer the duration of the plants receiving wastewater, the more the Fe plaque formed. The formation of Fe plaque could protect plants against waterlogging and increased their survival in anoxic environments (Armstrong, 1971). More Fe plaque formation was 
induced in mangrove roots receiving wastewater and heavy metals to immobilize the pollutants thus protecting plants against their toxicity (Pi et al., 2011; Mei et al., 2012). Plant species with more Fe plaque formation on root surfaces were more tolerant and more preferable in constructed wetlands for wastewater treatment (Mei et al., 2014). When compared E. agallocha and K. obovata, more Fe plaque was formed on root surfaces of the former species under tidal regimes with more flushing frequency (Te and To), but the difference was not significant under less frequent tidal flushing regimes ( $\mathrm{Tt}$ and $\mathrm{Tn}$ ). This indicated that E. agallocha was more tolerant to wastewater stress and waterlogging than $K$. obovata.

Significant negative relationships were found between the formation of Fe plaque on root surfaces and the total rate of ROL along a root during the 8-month irrigation with wastewater containing PAHs and PBDEs under all tidal treatments in both plant species (Fig. 5 and Table S2). Similar negative relationship between Fe plaque formation and ROL around rhizosphere of Lobelia dortmanna was also reported by Møller and Sand-Jensen that Fe plaque acted as a 'barrier' preventing oxygen released from the root leading to lower ROL (Moller and Sand-Jensen, 2008). In constructed wetlands, wastewater addition significantly decreased ROL but induced more Fe plaque formation in various wetland plants, and these two parameters exhibited negative correlations under wastewater stress (Pi et al., 2010b; Mei et al., 2014; Yang et al., 2014). On the contrary, positive relationships were found in rice that more ROL released from the root led to a higher concentration of Fe plaque 
(Armstrong, 1967). Otte et al. concluded that ROL contributed to the formation of Fe plaque on root surfaces in most wetland plants under waterlogged condition (Otte et al., 1991). The interaction between the rate of ROL and the concentration of Fe plaque formation in mangrove plants changed with time and wastewater treatment, with positive correlations in the control (without wastewater) and at the initial phase of the experiment, but changed to negative corelations afterwards and also in plants receiving strong wastewater (Pi et al., 2010b). Their study, together with the present findings on E. agallocha and K. obovata (Fig. 5 and Table S2), indicated that the formation of excess Fe plaque on root surfaces would function as a 'barrier' to prevent ROL in mangrove plants.

In both mangrove species at the same sampling time, regression relationships between the two treatments with more frequent tidal flushing (Te and To) followed the same trend which could be grouped together, while the two less frequent flushing treatments (Tt and Tn) formed another group (Fig. 5). The regression coefficient (B) between Fe plaque and ROL in the second group ( $\mathrm{Tt}$ and $\mathrm{Tn}$ ) were significantly higher than that in Te and To group (Table S2). Between the two plant species, B values of K. obovata were higher than that of E. agallocha, indicating the 'barrier' effect of Fe plaque on root ROL was more obvious in $K$. obovata (Table S2). This may be related to the weaker outer layers in roots of $K$. obovata than that in $E$. agallocha, thus required more barrier to prevent ROL (Pi et al., 2009). 
400 401 et al., 1995). It is because only soluble $\mathrm{Fe}^{2+}$ could be drawn by the transpiration 402 stream to reach plant root surfaces, spontaneously oxidized by root released $\mathrm{O}_{2}$, and

potential of soil (Eh value) relating to the concentrations of $\mathrm{Fe}^{2+}$ in soil (Mendelssohn then precipitated as Fe plaque (St-Cyr and Crowder, 1989; Chen et al., 2008). The duration and frequency of tidal flushing could affect the mobility of $\mathrm{Fe}^{2+}$ in soil and the amount of $\mathrm{O}_{2}$ around root surface (ROL) to oxidize $\mathrm{Fe}^{2+}$ (St-Cyr et al., 1993). It has been shown that submerged rice formed more Fe plaque on roots than the plants grown in aerobic soil, as the decrease of soil redox potential during flooding would lead to an increase of $\mathrm{Fe}^{2+}$ concentration in soil and larger amounts of dissolved $\mathrm{Fe}^{2+}$ would be moved to rhizosphere and oxidized by ROL to form Fe plaque (Liang et al., 2006; Chen et al., 2008). In aerobic soils, the mobility of $\mathrm{Fe}^{2+}$ may have decreased because of its precipitation and adsorption onto the bulk soil (Kirk, 2004). Similarly, Mendelssohn et al. also found that the exchangeable $\mathrm{Fe}$ in soils increased significantly during flooding leading to more Fe plaque formed on root surfaces (Mendelssohn et al., 1995).

The relationship between Fe plaque formation and soil redox potential was elucidated in several studies, suggesting that the concentration of Fe plaque formed on root surfaces was negatively correlated with the Eh value of the soil (Mendelssohn et al., 1995; Pi et al., 2010b, 2011). Similar findings were also obtained in the present study that more Fe plaque was formed in plants subject to more frequent tidal flushing (from Tn and Tt to To and Te) and longer duration of wastewater discharge 
421 (from 4- to 8-month) (Fig. 4). The Te- and To-treated soils had more negative redox

422 potential, and the Eh values decreased significantly from 4- to 8-month in all 423 treatments. The Eh values from 4- to 8-month in Te-, To-, Tt- and Tn-treated soil were $424-120.7$ to $-160.0 \mathrm{mV},-98.3$ to $-115.7 \mathrm{mV}, 135.4$ to $112.2 \mathrm{mV}$ and 157.7 to 134.3 , 425 respectively.

\section{Conclusions} surfaces of two mangrove plants, K. obovata and E. agallocha, continuously received wastewater containing PAHs and PBDEs, two types of very toxic and persistent formed, the less the $\mathrm{O}_{2}$ released from roots. The increase in the frequency of tidal flushing regimes decreased the 'barrier' effects of Fe plaque on ROL. The 'barrier' 
442 effect of Fe plaque on ROL in K. obovata with weaker root outer layers was more 443 significant than that in E. agallocha having stronger outer layers. These results 444 revealed that tidal flushing regimes significantly affected mangrove roots, especially 445 in K. obovata, the more sensitive species to tidal flushing, with root damages and 446 even death when grown in waterlogged soils continuously receiving wastewater447 borne PAHs and PBDEs.

\section{Acknowledgements}

450 The work described in this paper was financially supported by a City University of 451 Hong Kong (Project No.7004394 and 9680078) and a National Science Foundation grant from Mainland China (Project No. 41576086).

\section{References}

Armstrong, J., Armstrong, W., 2005. Rice: sulfide-induced barriers to root radial oxygen loss, $\mathrm{Fe}^{2+}$ and water uptake, and lateral root emergence. Ann. Bot. 96, 625638.

459 Armstrong, W., 1967. The oxidizing activity of roots in waterlogged soils. Plant Physiol. 20, 540-543. 
distance from apex, respiration and waterlogging. Plant Physiol. 25, 192-197.

Armstrong, W., 1979. Aeration in higher plants, in: Woolhouse, H.W. (Ed.), Advances in Botanical Research. Academic Press, London, pp. 225-332.

Armstrong, W., 1994. Polarographic oxygen electrodes and their use in plant aeration studies. Proceedings of the Royal Society of Edinburgh 102B, 511-527.

Armstrong, W., Strange, M.E., Cringle, S., Beckett, P.M., 1994. Microelectrode and modelling study of oxygen distribution in roots. Ann. Bot. 74, 287-299.

Binelli, A., Sarkar, S.K., Chatterjee, M., Riva, C., Parolini, M., Bhattacharya, B., Bhattacharya, A.K., Satpathy, K.K., 2008. A comparison of sediment quality guidelines for toxicity assessment in the Sunderban wetlands (Bay of Bengal, India). Chemosphere 73, 1129-1137.

Bodin, N., N'Gom Ka, R., Le Loc'h, F., Raffray, J., Budzinski, H., Peluhet, L., Tito de Morais, L., 2011. Are exploited mangrove molluscs exposed to Persistent Organic Pollutant contamination in Senegal, West Africa? Chemosphere 84, 318-327.

Branchi, I., Alleva, E., Costa, L.G., 2002. Effects of perinatal exposure to a 
484 485

486

487

488

489

490

491

492

493

494

495

496

497

498

499

500

501

502

503

504

polybrominated diphenyl ether (PBDE 99) on mouse neurobehavioural development.

Neurotoxicology 23, 375-384.

Brix, H., 1997. Do macrophytes play a role in constructed treatment wetlands? Water Sci. Technol. 35, 11-17.

Cao, Q.M., Chen, G.Z., Wang, H., Qin, J.Q., Huang, X.F., 2009. Distribution and sources of PAHs in surface sediments of Shantou mangrove wetlands, China. Fresen. Environ. Bull. 18, 1788-1797.

Chen, X.P., Kong, W.D., He, J.Z., Liu, W.J., Smith, S.E., Smith, F.A., Zhu, Y.G., 2008. Do water regimes affect iron-plaque formation and microbial communities in the rhizosphere of paddy rice? J. Plant Nutr. Soil Sci. 171, 193-199.

Cheng, H., Tam, N.F.Y., Wang, Y.S., Li, S.Y., Chen, G.Z., Ye, Z.H., 2012. Effects of copper on growth, radial oxygen loss and root permeability of seedlings of the mangroves Bruguiera gymnorrhiza and Rhizophora stylosa. Plant Soil 359, 255-266.

Cheng, H., Wang, Y.S., Fei, J., Jiang, Z.Y., Ye, Z.H., 2015. Differences in root aeration, iron plaque formation and waterlogging tolerance in six mangroves along a continues tidal gradient. Ecotoxicology 24, 1659-1667. 
506 Clarke, B.O., Porter, N.A., Symons, R.K., Marriott, P.J., Stevenson, G.J., Blackbeard, 507 J.R., 2010. Investigating the distribution of polybrominated diphenyl ethers through 508 an Australian wastewater treatment plant. Sci. Total Environ. 408, 1604-1611.

Colmer, T.D., 2003. Long-distance transport of gases in plants: a perspective on

511 internal aeration and radial oxygen loss from roots. Plant Cell Environ. 26, 17-36.

Darnerud, P.O., 2003. Toxic effects of brominated flame retardants in man and in wildlife. Environ. Int. 29, 841-853.

Dsikowitzky, L., Nordhaus, I., Jennerjahn, T.C., Khrycheva, P., Sivatharshan, Y., sediments and benthic organisms of the mangrove-fringed Segara Anakan Lagoon, Java, Indonesia. Mar. Pollut. Bull. 62, 851-862.

Farias, C.O., Hamacher, C., Scofield, A.D.L., 2008. Origin and degradation of hydrocarbons in mangrove sediments (Rio de Janeiro, Brazil) contaminated by an oil spill. Org Geochem. 39, 289-307. 
root crude enzyme extracts: potential role of nitrate reductase $(\mathrm{NaR})$ and glutathione S-transferase (GST) in their debromination. Chemosphere 90, 1885-1892.

Ikonomou, M.G., Rayne, S., Addison, R.F., 2002. Exponential increases of the brominated flame retardants, polybrominated diphenyl ethers, in the Canadian Arctic from 1981 to 2000. Environ. Sci. Technol. 36, 1886-1892.

Jackson, M.B., Fenning, T.M., Jenkins, W., 1985. Aerenchyma (Gas-space) Formation in Adventitious Roots of Rice (Oryza sativa L.) is not Controlled by Ethylene or Small Partial Pressures of Oxygen. J. Exp. Bot. 36, 1566-1572.

Kanaly, R.A., Harayama, S., 2010. Advances in the field of high-molecular-weight polycyclic aromatic hydrocarbon biodegradation by bacteria. Microb. Biotechnol. 3, $136-164$.

Ke, L., Yu, K.S., Wong, Y.S., Tam, N.F., 2005. Spatial and vertical distribution of polycyclic aromatic hydrocarbons in mangrove sediments. Sci. Total. Environ. 340, 177-187.

Kelly, B.C., Ikonomou, M.G., Blair, J.D., Gobas, F.A., 2008. Bioaccumulation behaviour of polybrominated diphenyl ethers (PBDEs) in a Canadian Arctic marine 
food web. Sci. Total. Environ. 401, 60-72.

Kirk, G.J.D., 2004. The Biogeochemistry of Submerged Soils. John Wiley and Sons, Chichester, UK.

Li, C.H., Zhou, H.W., Wong, Y.S., Tam, N.F., 2009. Vertical distribution and anaerobic biodegradation of polycyclic aromatic hydrocarbons in mangrove sediments in Hong Kong, South China. Sci. Total. Environ. 407, 5772-5779.

Liang, Y., Zhu, Y.G., Xia, Y., Li, Z., Ma, Y., 2006. Iron plaque enhances phosphorus uptake by rice (Oryza sativa) growing under varying phosphorus and iron concentrations. Ann. Appl. Biol. 149, 305-312.

Liu, H., Weisman, D., Ye, Y.B., Cui, B., Huang, Y.H., Colon-Carmona, A., Wang, Z.H., 2009a. An oxidative stress response to polycyclic aromatic hydrocarbon exposure is rapid and complex in Arabidopsis thaliana. Plant Sci. 176, 375-382.

Liu, Y., Tam, N.F.Y., Yang, J.X., Pi, N., Wong, M.H., Ye, Z.H., 2009b. Mixed heavy metal tolerance and radial oxygen loss in mangrove seedlings. Mar. Pollut. Bull. 58, 1843-1849. 
Mei, X.Q., Wong, M.H., Yang, Y., Dong, H.Y., Qiu, R.L., Ye, Z.H., 2012. The effect of radial oxygen loss on arsenic tolerance and uptake in rice grains and straw. Environ. Pollut. 165, 109-117.

Mei, X.Q., Yang, Y., Tam, N.F., Wang, Y.W., Li, L., 2014. Roles of root porosity, radial oxygen loss, Fe plaque formation on nutrient removal and tolerance of wetland plants to domestic wastewater. Water Res. 50, 147-159.

Mendelssohn, I.A., Keiss, B.A., Wakeley, J.S., 1995. Factors controlling the formation of oxidised root channels: a review. Wetlands 15, 37-46.

Moller, C.L., Sand-Jensen, K., 2008. Iron plaques improve the oxygen supply to root meristems of the freshwater plant, Lobelia dortmanna. New Phytol. 179, 848-856.

Otte, M.L., Dekkers, M.J., Rozema, J., Broekman, R.A., 1991. Uptake of arsenic by Aster tripolium in relation to rhizosphere oxidation. Can. J. Bot. 69, 2670-2677.

Page, A.L., Miller, R.H., Keeney, D.R., 1992. Methods of Soil Analysis. ASA and SSSA Inc., Madison, Wisconsin, USA.

Peng, X., Tang, C., Yu, Y., Tan, J., Huang, Q., Wu, J., Chen, S., Mai, B., 2009. 
589

590

591

592

593

594

595

596

597

598

599

600

601

602

603

604

605

606

607

608

609

Concentrations, transport, fate, and releases of polybrominated diphenyl ethers in sewage treatment plants in the Pearl River Delta, South China. Environ. Int. 35, 303309.

Pi, N., Tam, N.F., Wong, M.H., 2010a. Effect of wastewater discharge on root anatomy and radial oxygen loss (ROL) patterns of three mangrove species in southern China. Int. J. Phytoremediat. 12, 468-486.

Pi, N., Tam, N.F., Wong, M.H., 2010b. Effects of wastewater discharge on formation of Fe plaque on root surface and radial oxygen loss of mangrove roots. Environ. Pollut. 158, 381-387.

Pi, N., Tam, N.F., Wong, M.H., 2011. Formation of iron plaque on mangrove roots receiving wastewater and its role in immobilization of wastewater-borne pollutants. Mar. Pollut. Bull. 63, 402-411.

Pi, N., Tam, N.F.Y., Wu, Y., Wong, M.H., 2009. Root anatomy and spatial pattern of radial oxygen loss of eight true mangrove species. Aquat. Bot. 90, 222-230.

Ponnamperuma, F.N., 1984. Effects of flooding on soils, in: Kozlowski, T.T. (Ed.),

Flooding and Plant Growth. Academic Press, London, pp. 9-45. 
611 Sanchez-Avila, J., Bonet, J., Velasco, G., Lacorte, S., 2009. Determination and 612 occurrence of phthalates, alkylphenols, bisphenol A, PBDEs, PCBs and PAHs in an 613 industrial sewage grid discharging to a Municipal Wastewater Treatment Plant. Sci. 614 Total Environ. 407, 4157-4167.

615

616 Song, M., Chu, S., Letcher, R.J., Seth, R., 2006. Fate, partitioning, and mass loading 617 of polybrominated diphenyl ethers (PBDEs) during the treatment processing of 618 municipal sewage. Environ. Sci. Technol. 40, 6241-6246.

619

620 St-Cyr, L., Crowder, A.A., 1989. Factors affecting iron plaque on the roots of 621 Phragmites australis (Cav) Trin ex Steudal. Plant Soil 116, 85-93.

622

623 St-Cyr, L., Fortin, D., Campbell, P.G.C., 1993. Microscopic observation of the iron 624 plaque of a submerged aquatic plant vallisneria americana michx. Aquat. Bot. 46, $625 \quad 155-167$.

626

627 Tam, N.F.Y., Wong, Y.S., 2000. Hong Kong Mangrove. City University of Hong 628 Kong Press, Tat Chee Avenue, Kowloon, Hong Kong. 629 630 Taylor, G.J., Crowder, A.A., 1983. Use of the DCB technique for extraction of 
631

632

633

634

635

636

637

638

639

640

641

642

643

644

645

646

647

648

649

650

hydrous iron oxides from roots of wetland plants. Am. J. Bot. 70, 1254-1257.

Toyama, T., Furukawa, T., Maeda, N., Inoue, D., Sei, K., Mori, K., Kikuchi, S., Ike, M., 2011. Accelerated biodegradation of pyrene and benzo[a]pyrene in the Phragmites australis rhizosphere by bacteria-root exudate interactions. Water Res. 45, 1629-1638.

Visser, E.J.W., Colmer, T.D., Blom, C.W.P.M., Voesenek, L.A.C.J., 2000. Changes in growth, porosity, and radial oxygen loss from adventitious roots of selected monoand dicotyledonous wetland species with contrasting types of aerenchyma. Plant Cell Environ. 23, 1237-1245.

Wang, X., Yao, H., Wong, M.H., Ye, Z., 2013. Dynamic changes in radial oxygen loss and iron plaque formation and their effects on $\mathrm{Cd}$ and As accumulation in rice (Oryza sativa L.). Environ. Geochem. Health 35, 779-788.

651 
652 Yang, J., Tam, N.F.Y., Ye, Z.H., 2014. Root porosity, radial oxygen loss and iron 653 plaque on roots of wetland plants in relation to zinc tolerance and accumulation. Plant 654 Soil 374, 815-828.

655

656 Ye, Y., Tam, N.F.Y., 2002. Growth and physiological responses of Kandelia candel 657 and Bruguiera gymnorrhiza to livestock wastewater. Hydrobiologia 479, 75-81.

658

659 Zar, J.H., 1999. Biostatistical Analysis. Prentice-Hall, Upper Saddle River, New 660 Jersey.

661

662 Zhu, H., Wang, Y., Wang, X., Luan, T., Tam, N.F., 2014. Intrinsic debromination 663 potential of polybrominated diphenyl ethers in different sediment slurries. Environ. 664 Sci. Technol. 48, 4724-4731.

665 666 


\section{Figure legends}

Fig. 1 Effect of tidal regimes on dry biomass of leaf, stem and root in mangrove plants receiving wastewater contaminated with PAHs and PBDEs at 4-month (4M) and 8-month (8M) of the experiment (0: initial biomass; Te: Everyday flushing, To: tidal flushing at alternative day, Tt: tidal flushing twice per month, Tn: No tidal flushing; mean of three replicates were shown)

Fig. 2 Effects of tidal regimes on patterns of radial oxygen loss (ROL) along a lateral root in mangrove plants receiving wastewater contaminated with PAHs and PBDEs at 4-month (4M) and 8-month (8M) of the experiment (Te: Everyday flushing, To: tidal flushing at alternative day, Tt: tidal flushing twice per month, Tn: No tidal flushing; mean and standard deviation of three replicates were shown)

Fig. 3 Effects of tidal regimes on total radial oxygen loss (ROL) along a lateral root in mangrove plants receiving wastewater contaminated with PAHs and PBDEs at 4-month (4M) and 8-month (8M) of the experiment (Te: Everyday flushing, To: tidal flushing at alternative day, Tt: tidal flushing twice per month, Tn: No tidal flushing; mean and standard deviation of three replicates were shown; different letters in each tidal flushing treatment indicated significant difference between 4 and 8 months at $\mathrm{p} \leq 0.05$ according to independent sample $T$-test) 
Fig. 4 Effects of tidal regime on formation of Fe plaque on root surface in mangrove plants receiving wastewater contaminated with PAHs and PBDEs at 4-month (4M) and 8-month $(8 \mathrm{M})$ of the experiment (Te: Everyday flushing, To: tidal flushing at alternative day, Tt: tidal flushing twice per month, Tn: No tidal flushing; mean and standard deviation of three replicates were shown; different letters in each tidal flushing treatment indicated significant difference between 4 and 8 months at $p \leq 0.05$ according to independent sample $T$-test)

Fig. 5 Relationship between the formation of Fe plaque on root surface and total ROL along a lateral root during the 8-month experiment (4M: 4-month, 8M: 8-month; Te: Everyday flushing, To: tidal flushing at alternative day, Tt: tidal flushing twice per month, Tn: No tidal flushing; $\mathrm{n}=12$; Significance of regression coefficient $\mathrm{B}$ and coefficient of regression determination $\mathrm{R}^{2}$ are summarized in Table S2) 


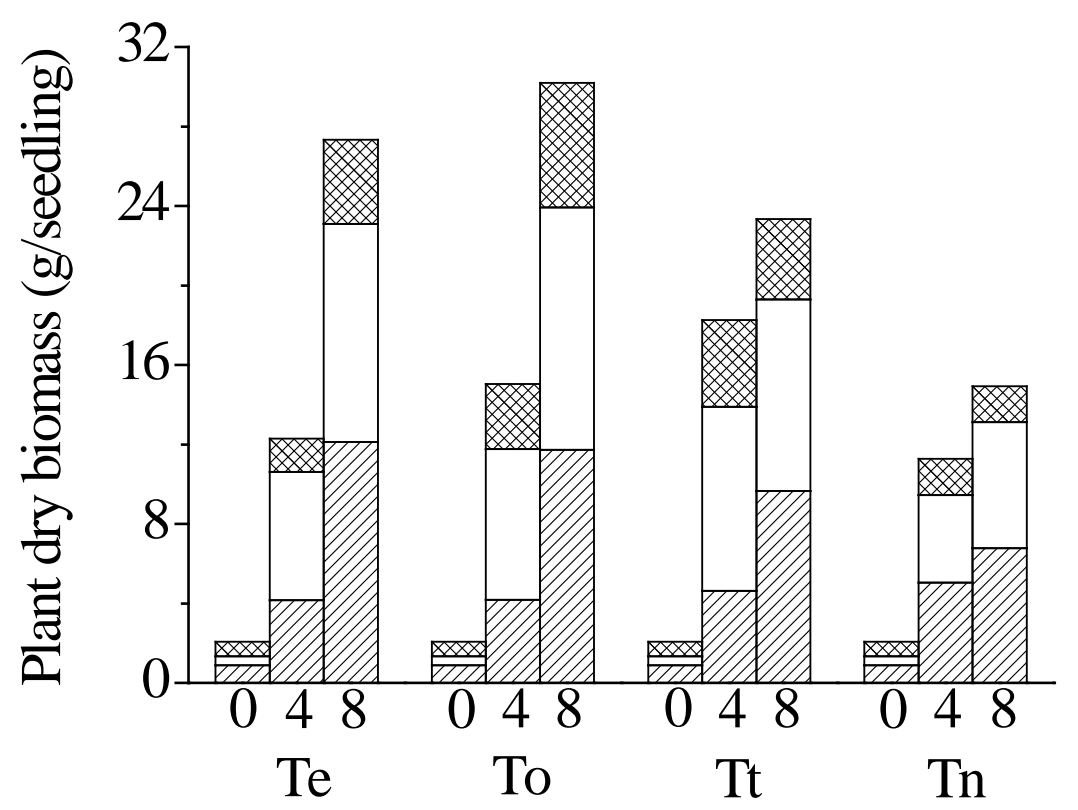

E. agallocha



K. obovata

Fig. 1 
(a) E. agallocha



Te

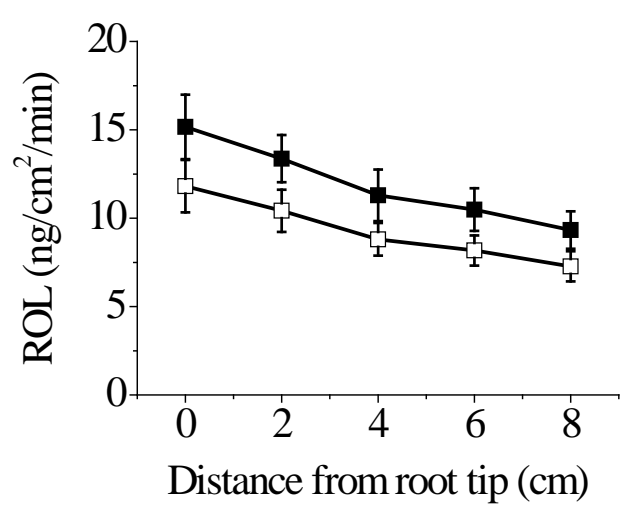

Te



To

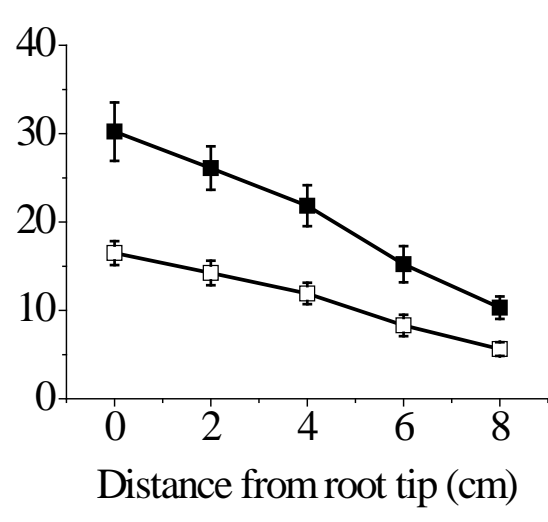

$\mathbf{T t}$

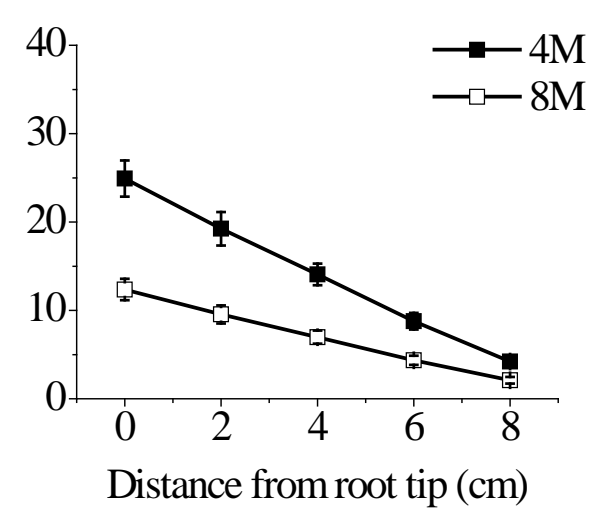

Tn

\section{(b) K. obovata}

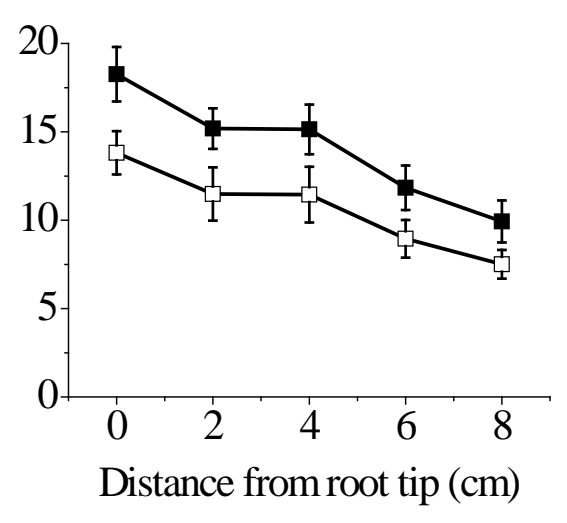

To

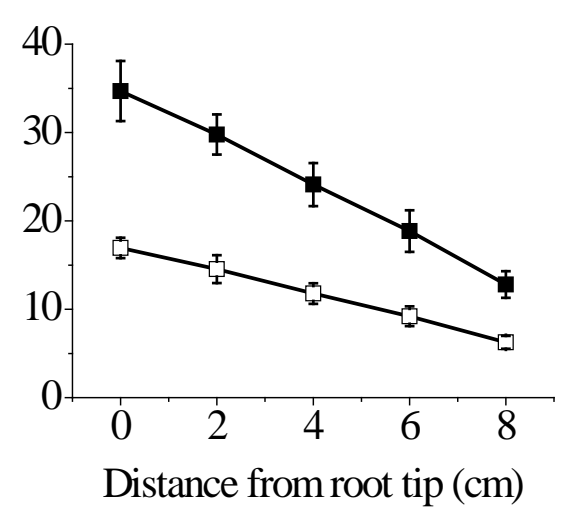

Tt

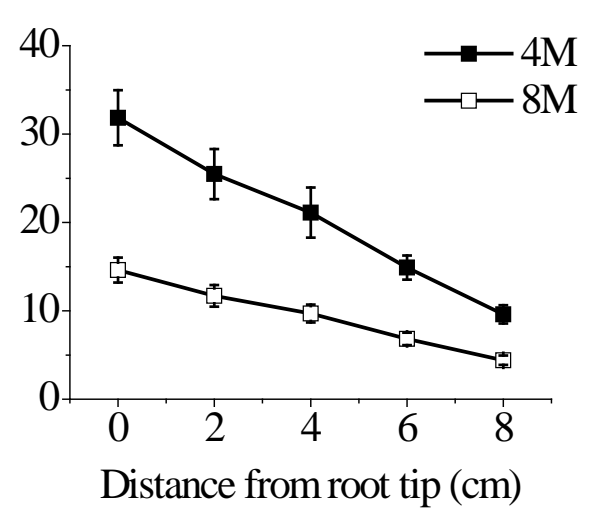

Tn

Fig. 2 




E. agallocha



K. obovata

Fig. 3 


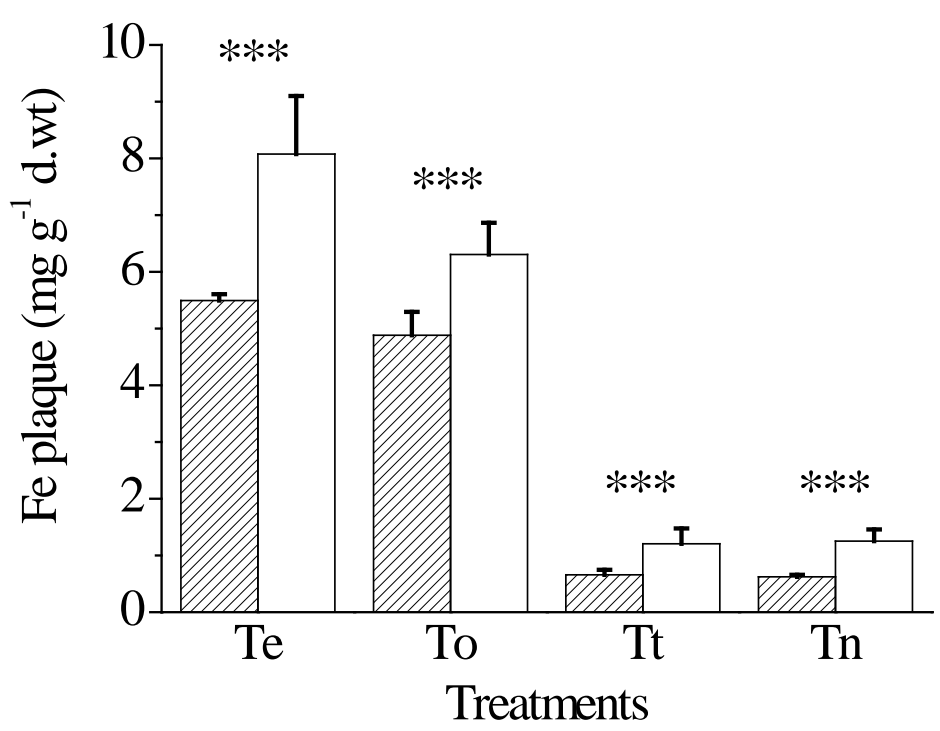

E. agallocha



K. obovata

Fig. 4 

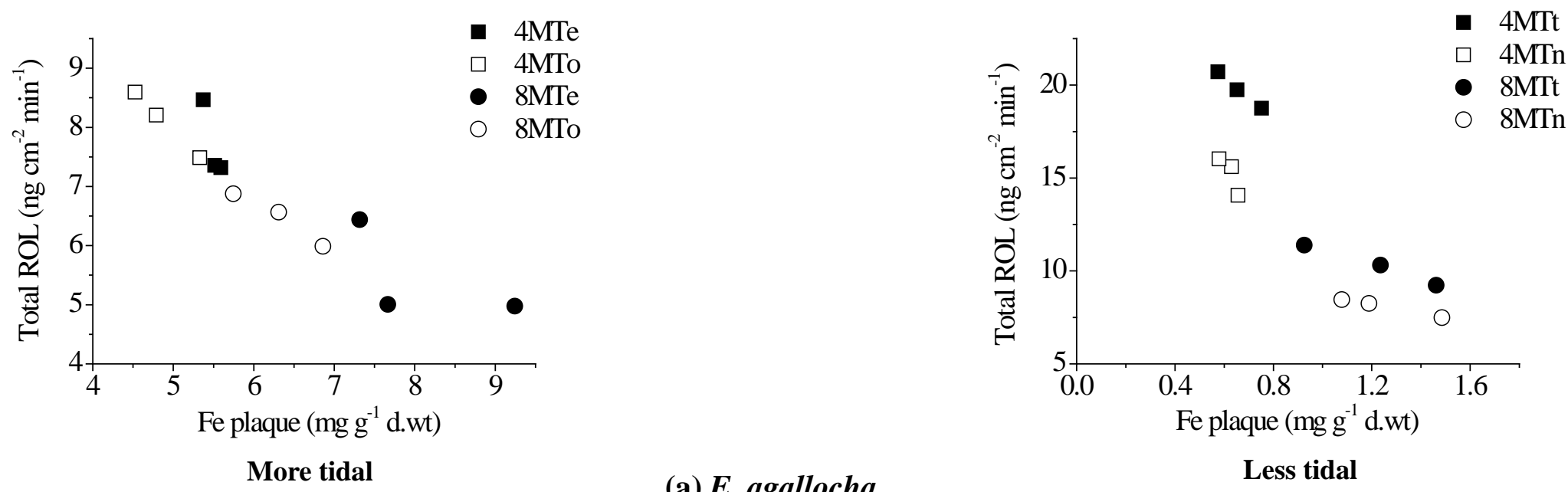

(a) E. agallocha

Less tidal
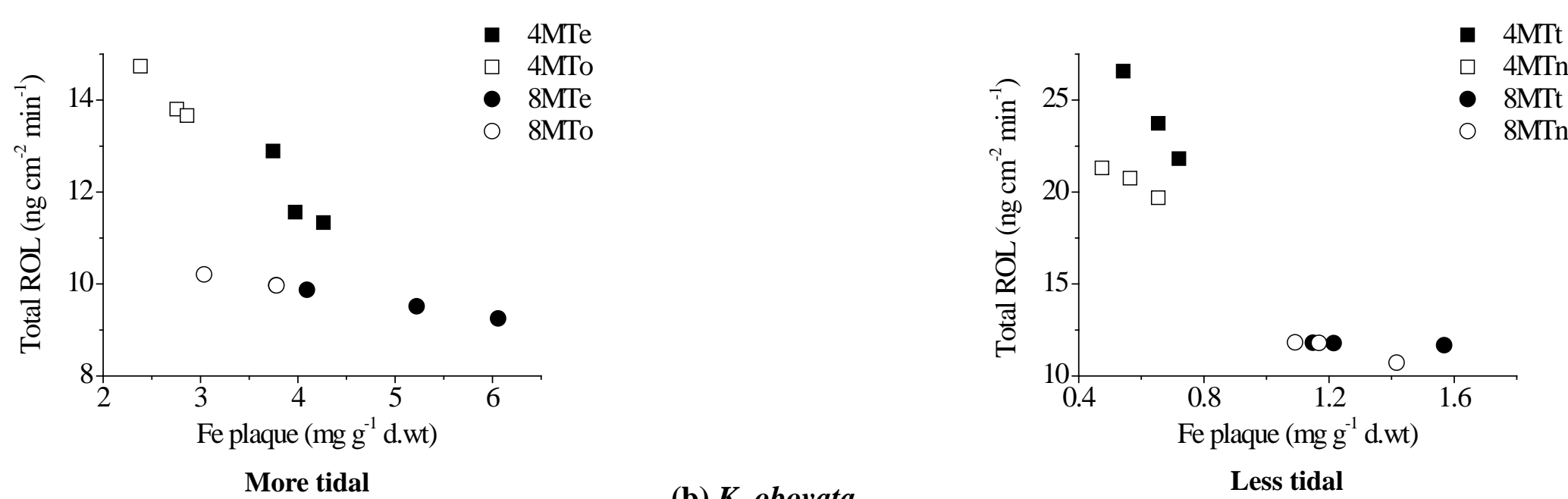

Fig. 5 
Table 1 Statistical analyses of the ANCOVA test, with time as the covariate, and species vs. tidal flushing regimes as the two factors, showing effects of mangrove plant species, tidal flushing regimes and sampling time on the plant dried biomass, ROL from roots, and Fe plaque formed on root surface $\left({ }^{*}: \mathrm{p}\right.$ $\leq 0.05, * *: \mathrm{p} \leq 0.01, * * *: \mathrm{p} \leq 0.001$, NS: not significant)

\section{Sources of variations}

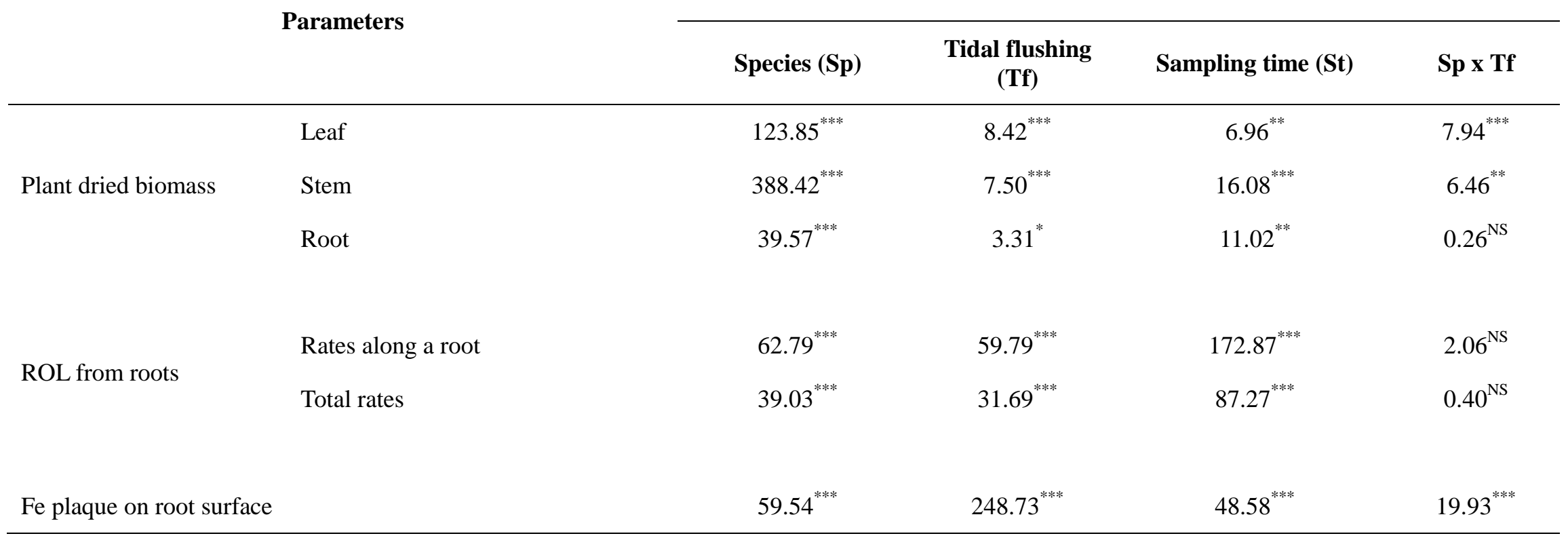

